\title{
Diagnostic value of transcranial ultrasonography for selecting subjects with large vessel occlusion: a systematic review
}

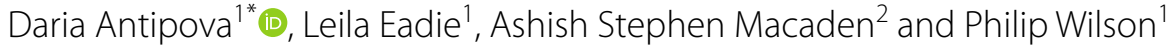

\begin{abstract}
Introduction: A number of pre-hospital clinical assessment tools have been developed to triage subjects with acute stroke due to large vessel occlusion (LVO) to a specialised endovascular centre, but their false negative rates remain high leading to inappropriate and costly emergency transfers. Transcranial ultrasonography may represent a valuable pre-hospital tool for selecting patients with LVO who could benefit from rapid transfer to a dedicated centre.

Methods: Diagnostic accuracy of transcranial ultrasonography in acute stroke was subjected to systematic review. Medline, Embase, PubMed, Scopus, and The Cochrane Library were searched. Published articles reporting diagnostic accuracy of transcranial ultrasonography in comparison to a reference imaging method were selected. Studies reporting estimates of diagnostic accuracy were included in the meta-analysis.

Results: Twenty-seven published articles were selected for the systematic review. Transcranial Doppler findings, such as absent or diminished blood flow signal in a major cerebral artery and asymmetry index $\geq 21 \%$ were shown to be suggestive of LVO. It demonstrated sensitivity ranging from 68 to 100\% and specificity of 78-99\% for detecting acute steno-occlusive lesions. Area under the receiver operating characteristics curve was 0.91. Transcranial ultrasonography can also detect haemorrhagic foci, however, its application is largely restricted by lesion location.

Conclusions: Transcranial ultrasonography might potentially be used for the selection of subjects with acute LVO, to help streamline patient care and allow direct transfer to specialised endovascular centres. It can also assist in detecting haemorrhagic lesions in some cases, however, its applicability here is largely restricted. Additional research should optimize the scanning technique. Further work is required to demonstrate whether this diagnostic approach, possibly combined with clinical assessment, could be used at the pre-hospital stage to justify direct transfer to a regional thrombectomy centre in suitable cases.
\end{abstract}

Keywords: Large vessel occlusion, Stroke, Acute cerebral ischaemia, Intracerebral haemorrhage, Transcranial ultrasonography, Neuroimaging

\section{Introduction}

\section{Rationale}

Stroke is the second largest cause of death and one of the most common causes of complex disability in adults worldwide [1]. Mechanical thrombectomy (MT) preceded by intravenous thrombolysis provides optimal care for patients with acute ischaemic stroke due to large

\footnotetext{
*Correspondence: daria.antipova@abdn.ac.uk

${ }^{1}$ Centre for Rural Health, University of Aberdeen, Old Perth Road, Inverness IV2 3JH, UK

Full list of author information is available at the end of the article
}

vessel occlusion (LVO) [2]. While thrombolysis is offered in many general hospitals, MT can only be performed in specialised endovascular centres with neurointerventional facilities. Earlier initiation of endovascular reperfusion is associated with greatly improved outcome [3, 4]. Therefore, patients with suspected LVO could benefit from direct transfer to a specialised endovascular centre.

A number of clinical assessment tools have been developed to triage subjects, but their false negative rates remain high which means that more than $20 \%$ of patients with LVO would be transferred to a centre with no 
dedicated facilities available [5]. Furthermore, false positive results can lead to unnecessary and costly emergency transfers. Hence, there is an urgent need to establish a reliable process to select patients with LVO, particularly in areas with long transport times.

Portable transcranial ultrasound (see Glossary for explanations of the different ultrasound types) has emerged as a potential method for rapidly assessing intracranial circulation and brain structures in the prehospital and early hospital phase [6]. The evidence base for the diagnostic accuracy of transcranial ultrasonography in acute stroke population appears to be extensive, with more than 150 publications. Up to date, it has not been demonstrated whether ultrasonography can be implemented as part of a triage system for patients with LVO to streamline care. A systematic analysis of the existing data in the field was therefore performed.

\section{Objectives}

The following questions are addressed in the current review:

1. What is the diagnostic accuracy of transcranial ultrasonography in detecting occlusion and/or stenosis of cerebral vessels in acute ischaemic stroke population compared with other available diagnostic techniques?

2. Can transcranial ultrasonography be a useful tool to exclude intracranial haemorrhage as one of the contraindications for endovascular reperfusion compared with other available diagnostic techniques?

3. Can transcranial ultrasonography be a useful tool to detect midline shift suggestive of space-occupying stroke compared with other available diagnostic techniques?

\section{Methods}

\section{Protocol and registration}

The protocol is registered in PROSPERO, International prospective register of systematic reviews, and can be accessed at: https://www.crd.york.ac.uk/prospero/displ ay_record.php?RecordID $=75882$.

\section{Eligibility criteria}

Inclusion and exclusion criteria are listed in Table 1.

\section{Information sources}

A systematic search of the literature was conducted during May-June 2017, using a database-specific search strategy for each of the following electronic databases: PubMed, Medline, Embase, Scopus and The Cochrane Library.

\section{Search}

The search strategy included a combination of multiple iterations of $\mathrm{MeSH}$ and keyword terms relating to each component of the research question as outlined in Additional file 1.

The search was restricted to human studies, English language, and adult participants. There was no restriction for the year of publication.

\section{Study selection}

The study selection process is illustrated in Fig. 1.

Eligible papers were tabulated and used in the qualitative synthesis. Studies which reported diagnostic accuracy values such as true positive (TP), false positive (FP), true negative (TN), false negative (FN), and sensitivity and specificity values were included in the quantitative analysis.

\section{Data collection and extraction}

A dedicated data extraction form was developed and used to collect relevant information from the included studies. The inclusion of information fields in the data collection form was guided by the review questions. The following components were assessed:

- Study setting.

- Main characteristics of the patient population.

- Number of subjects recruited into the study and included in the study analysis.

- Ethical approval.

- Modality of transcranial ultrasonography being investigated, its technical considerations and qualifications of researchers performing transcranial ultrasound scanning.

- Reference test and technical considerations.

- Blindness of investigators performing and/or interpreting the tests' results.

- Time interval from symptom onset to index and reference tests, time interval between index and reference tests.

- Whether results were presented in the form of diagnostic accuracy (sensitivity, specificity, TP, FP, TN, FN, predictive values), or a narrative.

Two of this review's authors completed the data extraction form for each paper. If authors disagreed, a third author adjudicated. As our analysis concerned only published data, further data from investigators were not sought. 


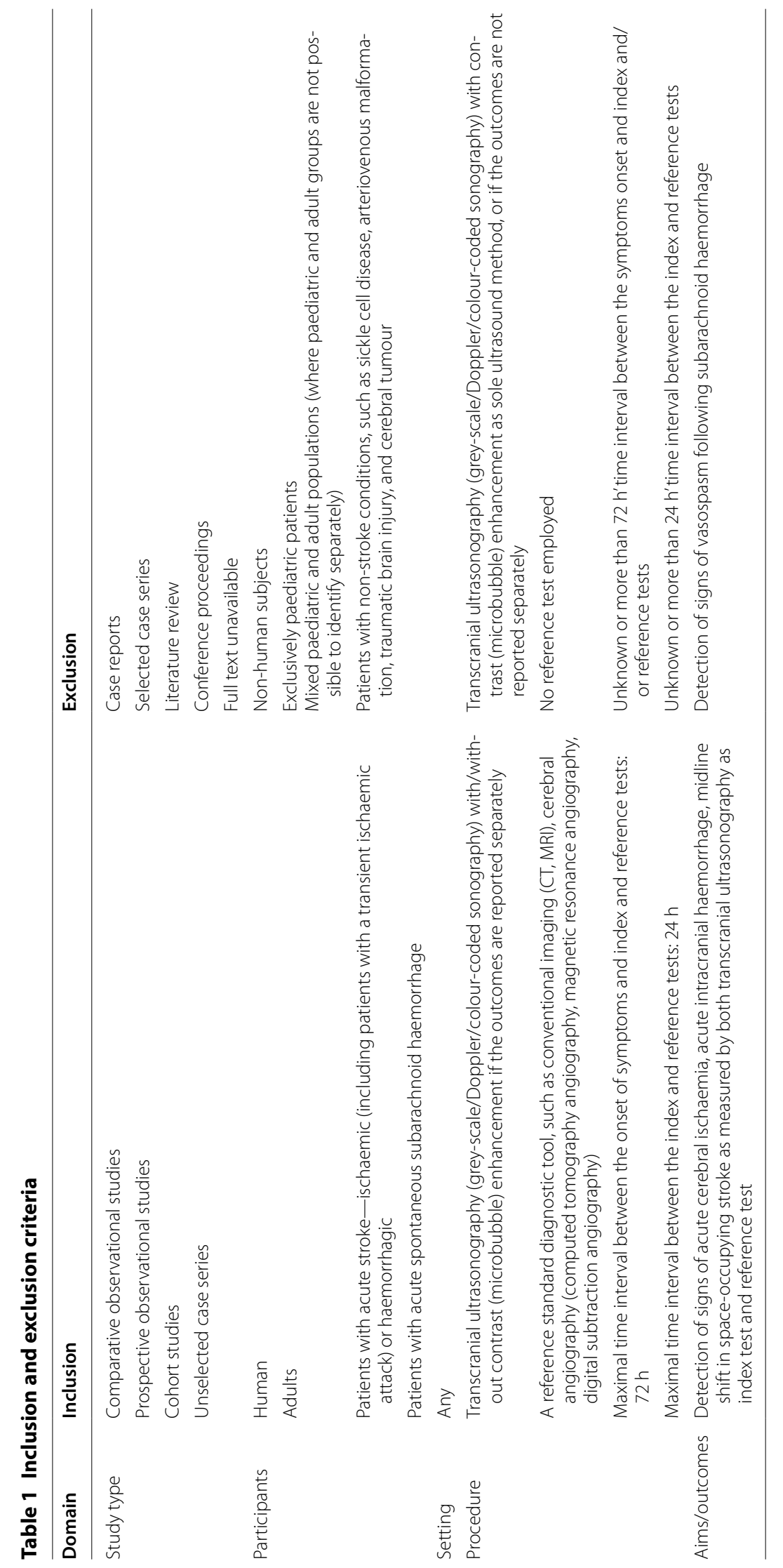




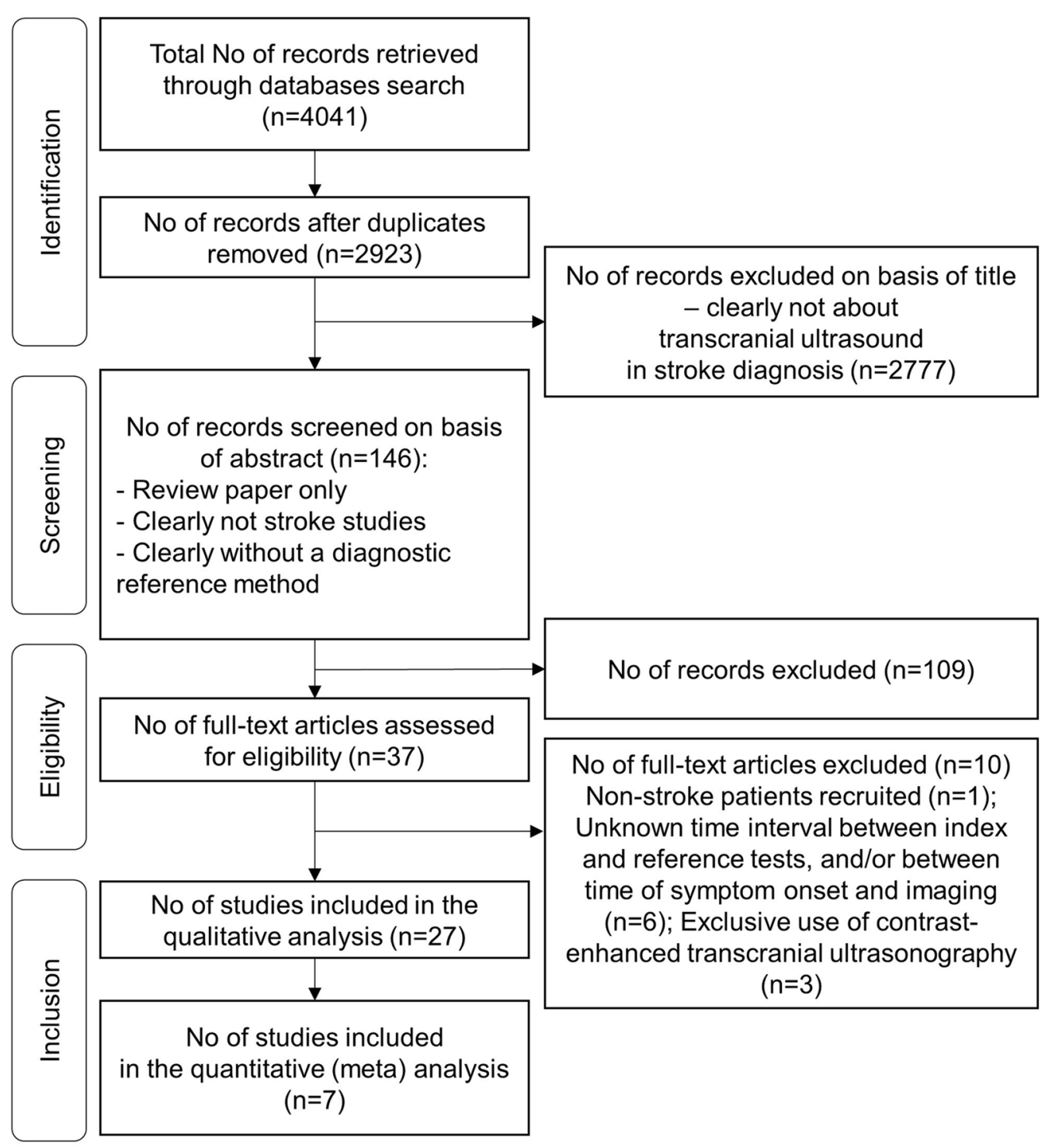

Fig. 1 PRISMA flowchart. Outline of the study selection process using inclusion and exclusion criteria

\section{Risk of bias assessment in individual studies}

Included studies were assessed qualitatively for concerns regarding their applicability for each of the four domains: patient selection, index test, reference standard, and flow and timing, in accordance with the QUADAS-2 Tool quality assessment system [7]. The risk of bias and applicability concerns were identified using RevMan 5.3 software and presented as a summary table.

\section{Statistical analysis}

The statistical analysis was performed in accordance with the Cochrane guidelines for diagnostic test accuracy reviews [8]. Two-by-two tables were constructed for the transcranial ultrasound results of diagnosis of acute steno-occlusion, intracranial haemorrhage, and detection of midline shift showing the binary test results cross-classified with the binary reference standard. The diagnostic values including the TP, TN, FP, and FN values were entered into RevMan 5.3. The sensitivity and specificity, as well as their $95 \%$ confidence intervals, were also calculated in RevMan 5.3. The data from each study were presented graphically by plotting sensitivities and specificities on a coupled forest plot and summary receiver operating characteristics (SROC) plot. Area under the curve for the SROC plot was calculated as a summary 
value for given sensitivities and specificities using IBM SPSS Statistics 25 package.

\section{Risk of bias assessment across studies}

Provided that the number of studies reporting diagnostic accuracy values was ten or above, publication bias assessment with a funnel plot to illustrate the possibility of selective publication of small studies with positive results would be performed.

\section{Results}

\section{Study selection}

The results of the study selection process are illustrated in Fig. 1.

\section{Study characteristics Included studies}

27 studies in which participants were subjected to both transcranial ultrasonography and gold standard diagnostic imaging for diagnosing acute stroke were eligible for inclusion in the final analysis. In all studies, transcranial ultrasound was performed within $72 \mathrm{~h}$ of stroke symptom onset. Time interval between the index and reference tests did not exceed $24 \mathrm{~h}$. Basic characteristics of included papers are presented in Table 2 .

All papers described prospective observational studies. In total, 1683 participants (patients $n=1566$, controls $n=117$ ) were analysed across the 27 study populations. Individual studies varied considerably in sample size, from 4 to 213 subjects, and consisted of participants recruited upon presentation to the hospital or outpatient departments. There were no pre-hospital studies identified that would meet the inclusion criteria for the current systematic review.

The search did not retrieve any eligible studies aimed to assess accuracy of transcranial ultrasonography for detecting signs of subarachnoid haemorrhage (SAH).

Seven papers presented study results reporting quantitative values of the diagnostic accuracy of the transcranial ultrasonography (TP, TN, FP, FN, sensitivity, and specificity), whereas 20 discussed outcomes qualitatively or did not fully report diagnostic accuracy values.

On the basis of their full-text articles, ten studies were not included in the final analysis [9-18]. Principal reasons were unknown or unclear time interval between symptom onset and reference/index imaging and/or between reference and index imaging $[9,10$, 12, 14-18], the use of contrast-enhanced transcranial sonography either exclusively or in combination with non-contrast ultrasound without reporting outcomes separately for each group $[9,11,13]$, or a non-stroke population [12].

\section{Risk of bias assessment in individual studies}

A summary of bias and applicability concerns is presented in Table 3.

Case-control methodologies were employed in three included studies [19-21] in which groups of patients with and without the target condition were identified before the index test was performed.

The papers were also evaluated in regard to the sample size and divided into three groups [22]: low sample size ( $<100$ participants), intermediate (100-300 participants), and high ( $>300$ participants). Only six studies had intermediate sample size, whereas in the remaining 21 it was low.

Ethical approval was not reported in $44 \%(n=12)$ of the included papers.

A list of transcranial ultrasonography modalities used in individual studies as an index test is provided in Table 2. All included studies were assessed against the adequate blinding criterion. In 19, the results of the index test were interpreted without knowledge of the results of the reference standard [19, 23-40]. Two studies $[41,42]$ were lacking blindness, and in 6 papers it was not explicitly mentioned whether or not the investigator was blinded to the results of the reference standard [20, 21, 43-46].

Studies in which transcranial ultrasound scanning and reference standards were performed on the same patient group and the whole sample should have received verification of the diagnosis by the reference standard were included. The control methods are listed in Table 2.

Although all studies that did not employ a reference imaging modality were excluded, there was inconsistency in application and interpretation of the various reference standard tests. Some of the studies used several imaging modalities as a reference test, and for most articles, it was either computed tomography angiography (CTA), magnetic resonance angiography (MRA), or digital subtraction angiography (DSA) [21, 25, 27, 39]. For example, in the paper by Gerriets et al. [27], the choice of reference test (CTA, MRA, or DSA) was left up to the treating centre.

\section{Diagnostic accuracy of transcranial ultrasonography in acute stroke}

The diagnostic accuracy of transcranial ultrasonography in detecting signs of acute steno-occlusive lesions, intracranial haemorrhage and/or midline shift was a primary outcome in all studies. All included studies that specified the type of a probe and frequency of transcranial ultrasonography performed scanning with a sector probe at low frequency (2-4 MHz) (Table 2). Kenton et al. [29] used a curved phase array probe for their study, however, 


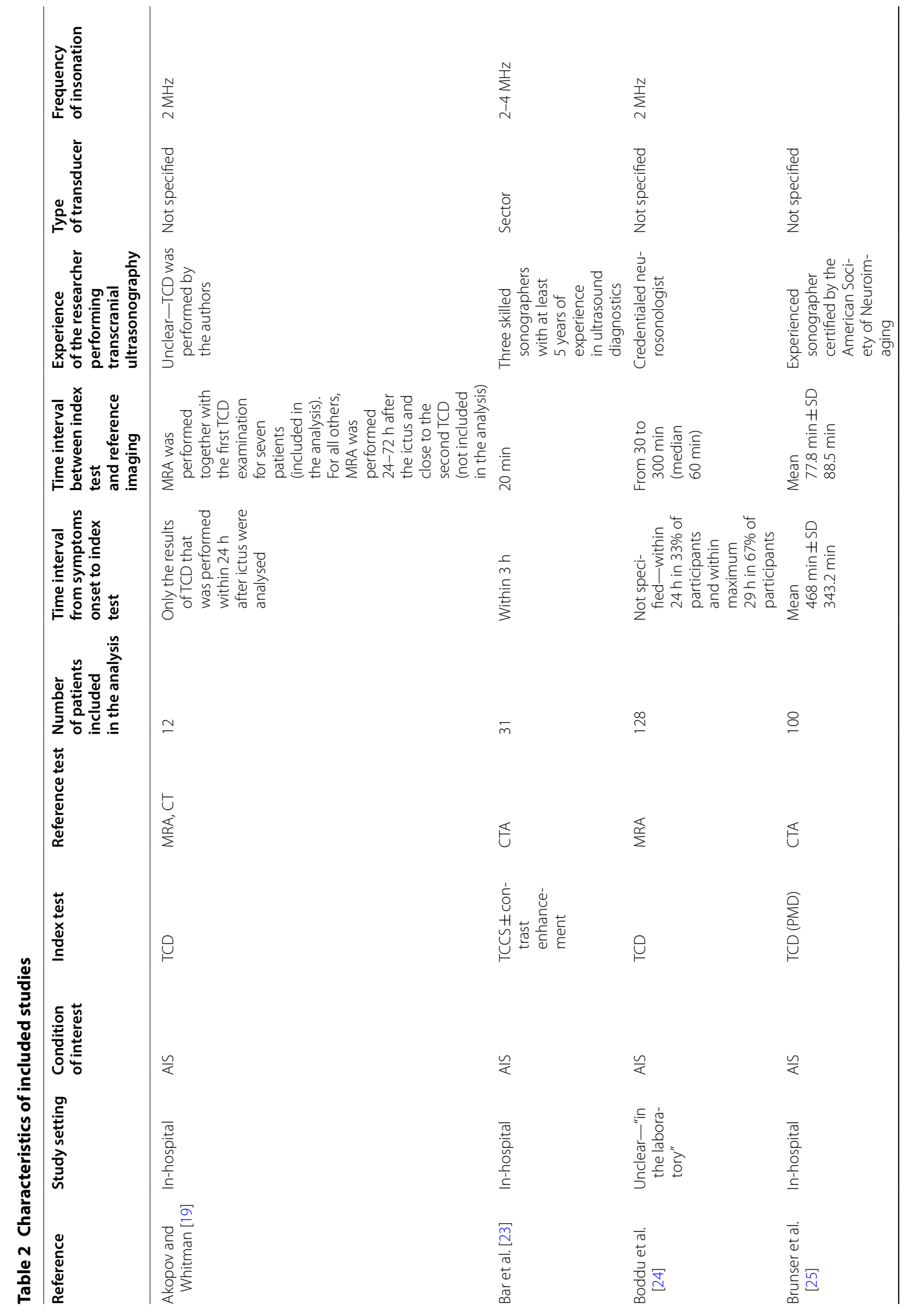




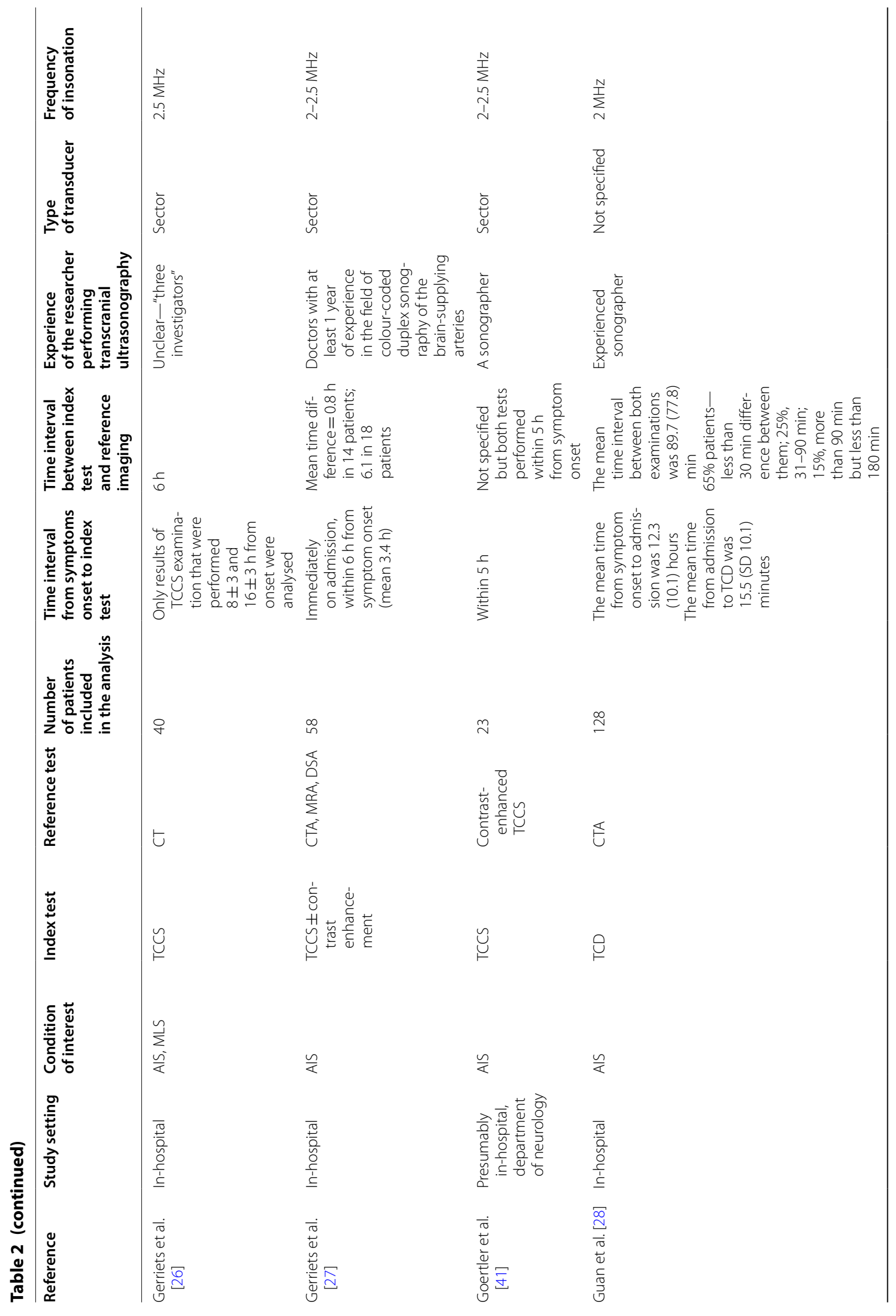




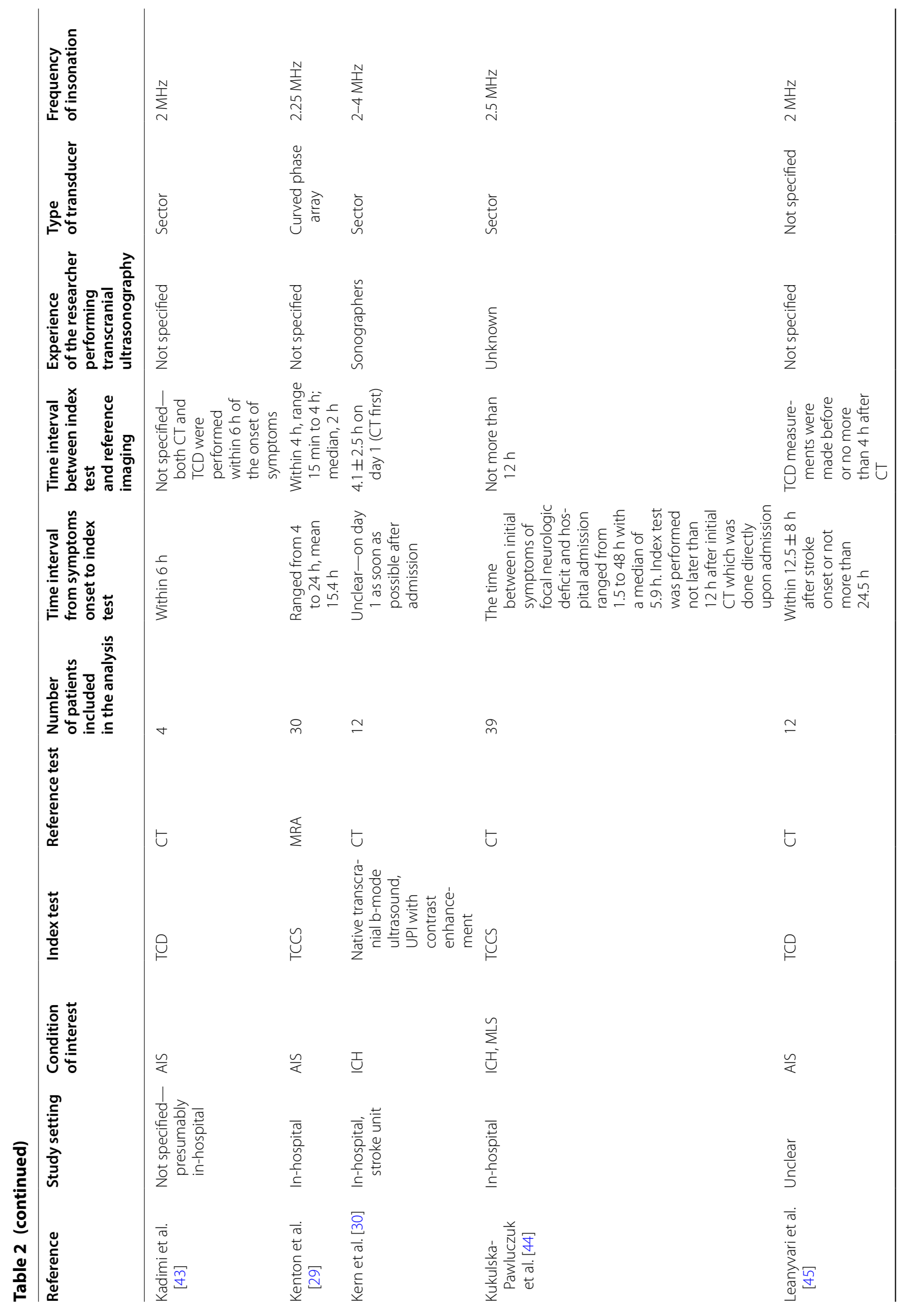




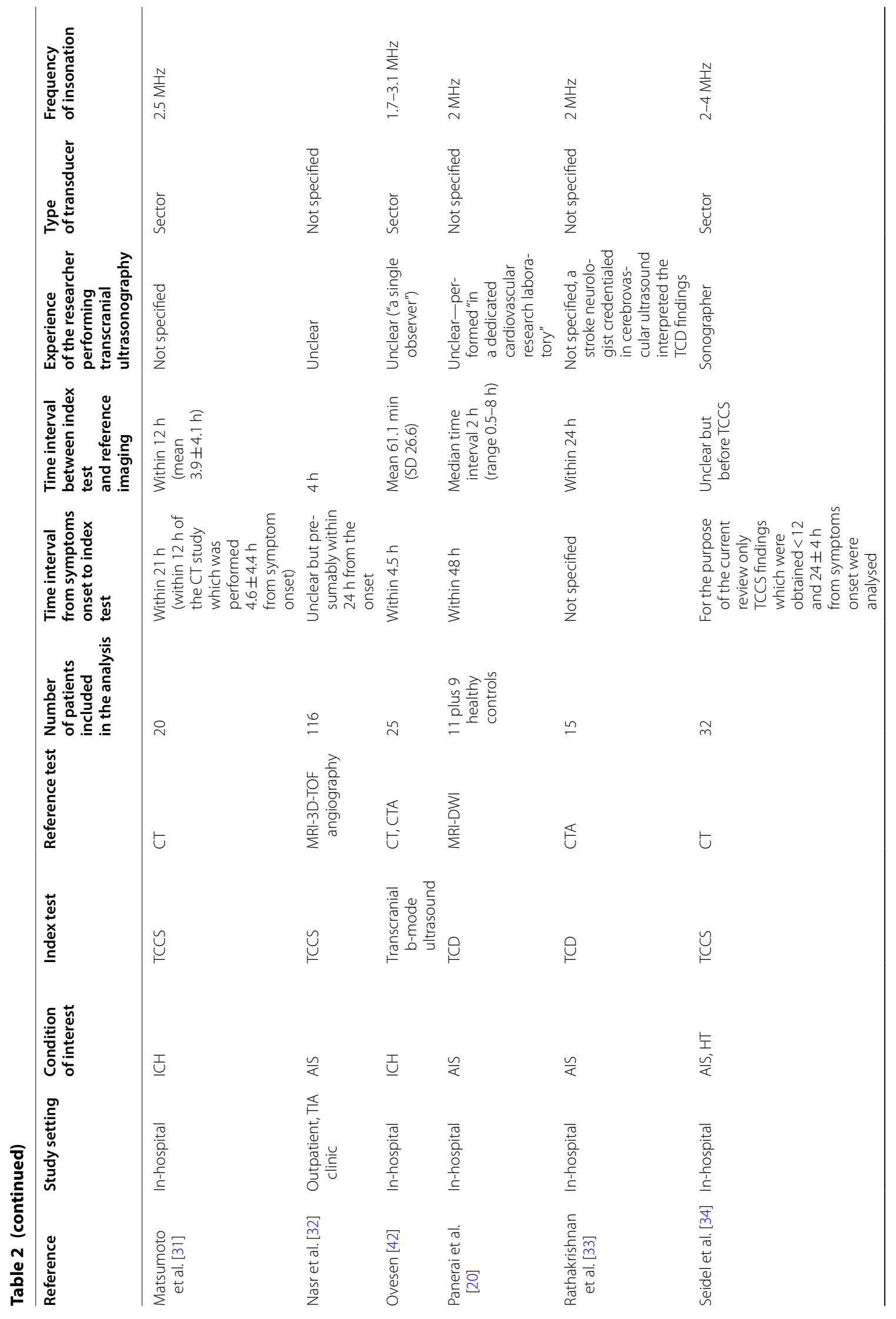




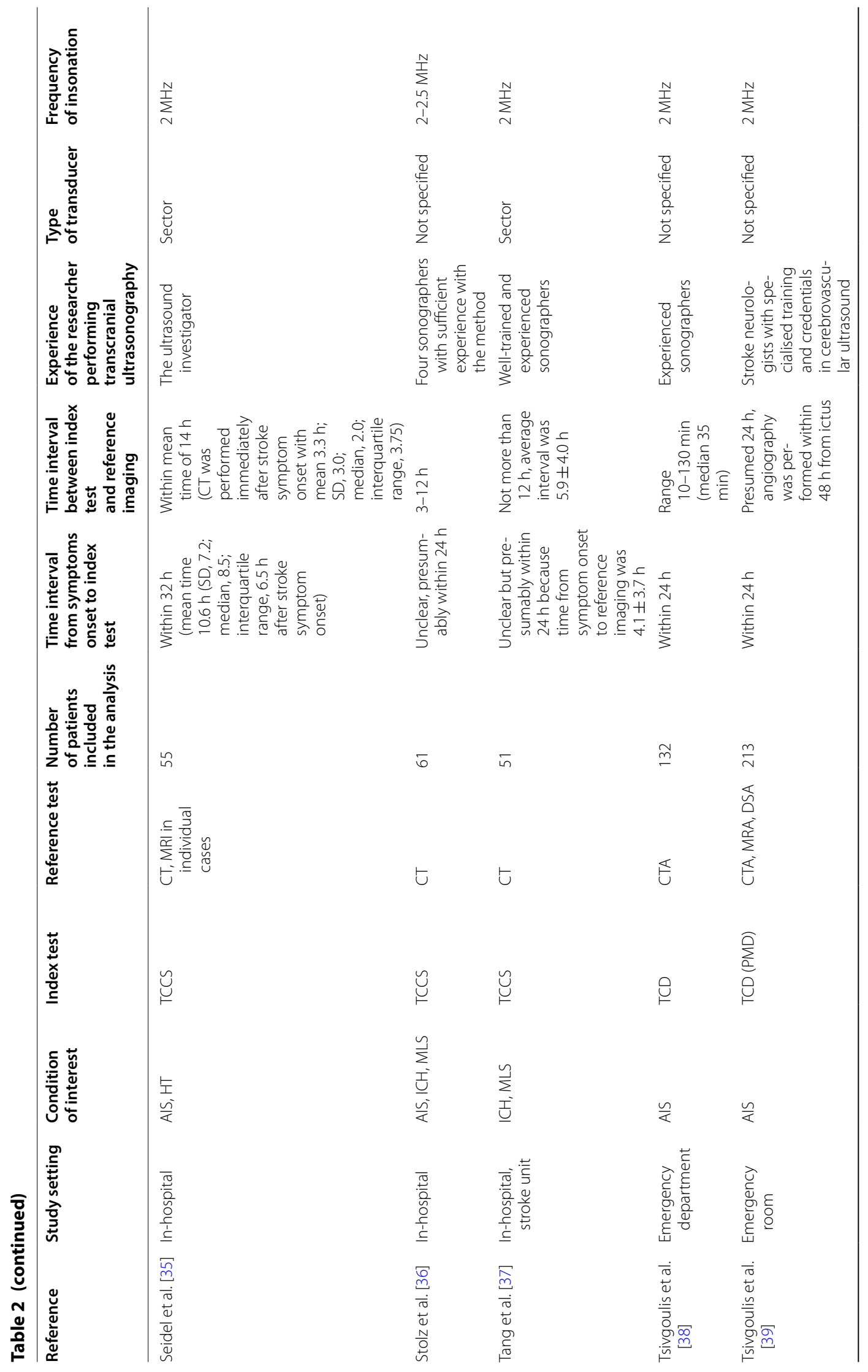




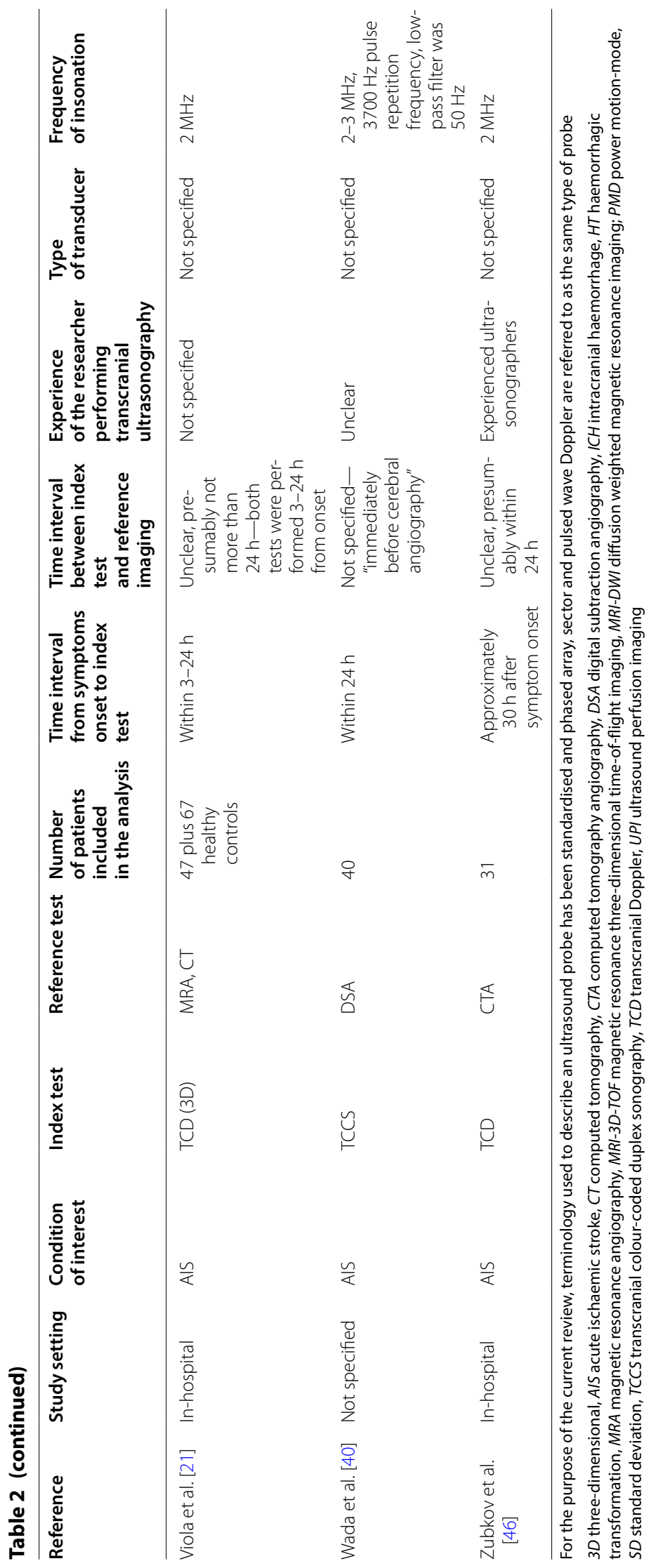


Table 3 Risk of bias and applicability concerns summary

\begin{tabular}{|c|c|c|c|c|c|c|c|}
\hline & \multicolumn{4}{|c|}{ Risk of bias } & \multicolumn{3}{|c|}{ Applicability concerns } \\
\hline & $\begin{array}{l}\text { Patient } \\
\text { selection }\end{array}$ & Index test & $\begin{array}{l}\text { Reference } \\
\text { standard }\end{array}$ & Flow and timing & Patient selection & Index test & $\begin{array}{l}\text { Reference } \\
\text { standard }\end{array}$ \\
\hline Akopov and Whitman [19] & High & Low & Unclear & Low & Low & Low & Low \\
\hline Bar et al. [23] ${ }^{a}$ & Low & Low & Low & High & Low & Low & Low \\
\hline Boddu et al. [24] & Low & Low & Low & Low & Low & Low & Low \\
\hline Brunser et al. [25] $]^{\mathrm{a}}$ & Low & Low & Low & Unclear & Low & Low & Low \\
\hline Gerriets et al. [26] & Low & Low & Low & Low & Low & Low & Low \\
\hline Gerriets et al. [27] & Low & Low & Low & High & Low & Low & Low \\
\hline Goertler et al. [41] & Unclear & High & High & Unclear & High & Low & Low \\
\hline Guan et al. [28] ${ }^{\mathrm{a}}$ & Unclear & Low & Low & Low & Unclear & Low & Low \\
\hline Kadimi et al. [43] & Unclear & Unclear & Unclear & Low & Low & Low & Low \\
\hline Kenton et al. [29] & Unclear & Low & Low & High & Low & Low & Low \\
\hline Kern et al. [30] & Low & Low & Low & Low & Low & Low & Low \\
\hline $\begin{array}{l}\text { Kukulska-Pawluczuk et al. } \\
\text { [44] }\end{array}$ & Unclear & Unclear & Low & High & Low & Low & Low \\
\hline Leanyvari et al. [45] & High & Low & High & Low & Low & Low & High \\
\hline Matsumoto et al. [31] & Low & Low & Low & Unclear & Low & Low & Low \\
\hline Nasr et al. [32] & Low & Low & Low & High & Low & Low & Low \\
\hline Ovesen et al. [42] & Low & High & Low & High & Low & Low & Low \\
\hline Panerai et al. [20] & High & High & High & Low & High & Low & High \\
\hline Rathakrishnan et al. [33] ${ }^{a}$ & High & Low & Low & Unclear & Low & Low & Low \\
\hline Seidel et al. [34] & High & Low & Unclear & Low & Low & Low & Low \\
\hline Seidel et al. [35] & Unclear & Low & Unclear & Low & Low & Low & Low \\
\hline Stolz et al. [36] & Unclear & Low & Unclear & Low & Low & Low & Low \\
\hline Tang et al. [37] & Low & Low & Unclear & High & Low & Low & Low \\
\hline Tsivgoulis et al. [38] & Low & Low & Low & Low & Low & Low & Low \\
\hline Tsivgoulis et al. [39] & Unclear & Low & Low & Low & Low & Low & Low \\
\hline Viola et al. [21] & High & Unclear & High & High & Low & Low & High \\
\hline Wada et al. [40] ${ }^{a}$ & Low & Low & Low & Low & Low & Low & Low \\
\hline Zubkov et al. [46] & Unclear & High & Unclear & High & Low & Unclear & Low \\
\hline
\end{tabular}

Review author's judgement about each domain for each included study

Studies marked with an "al" were included in the quantitative analysis

this did not seem to provide any additional and/or contradictory information to other reviewed studies.

The majority of included papers (22/27) aimed to evaluate diagnostic accuracy of transcranial Doppler sonography (TCD) in detecting signs of acute occlusion and/or stenosis in acute ischaemic stroke population (Table 2). However, there was marked diversity in diagnostic protocol and criteria for steno-occlusive lesions (Additional files 2 and 3 ).

Seventeen studies assessed diagnostic value of TCD in identifying occlusion of the cerebral arteries $[19,21$, 23, 25, 27-29, 32, 33, 35, 38-41, 43, 45, 46]. Diagnosis of the middle cerebral artery (MCA) mainstem occlusion can be considered when the flow was absent or minimal, blunted, or damped throughout the MCA while the flow in the distal internal carotid artery, anterior and posterior cerebral arteries was diverted $[23,27,35,38,40]$. Similarly, anterior cerebral artery occlusion and occlusion in the posterior circulation (vertebral, basilar and posterior cerebral arteries) were defined as the absence of the flow or the presence of minimal, blunted, or dampened flow signals throughout these vessels $[38,39]$.

The interhemispheric asymmetry index has been used to describe the difference in the blood flow velocity between symptomatic and asymptomatic hemispheres [47]. Asymmetry index $\geq 21 \%$ was shown to correlate accurately with clinical presentation and to detect major branch or multiple branch occlusion [21, 23, 29, 35, 41]. In patients with an asymmetry index of $<21 \%$, conventional imaging (CT, MRI) is likely to offer normal appearance or show only very minor changes suggestive of acute brain ischemia [21, 28, 29]. Kadimi et al. [43] reported a 


\begin{tabular}{|c|c|c|c|c|c|c|c|c|}
\hline Study & $\mathrm{TP}$ & FP F & FN & IN & Sensitivity ( $(95 \% \mathrm{Cl})$ & Specificity ( $95 \%$ Cl) & Sensitivity (95\% Cl) & Specificity (95\% Cl) \\
\hline Bar 2010 & 22 & 2 & 0 & $?$ & $1.00[0.85,1.00]$ & $0.78[0.40,0.97]$ & & $=$ \\
\hline Brunser 2009 & 30 & 4 & 3 & 63 & $0.91[0.76,0.98]$ & $0.94[0.85,0.98]$ & $\rightarrow$ & $\rightarrow$ \\
\hline Guan 2013 & 35 & 1 & 0 & 92 & $1.00[0.90,1.00]$ & $0.99[0.94,1.00]$ & $\rightarrow$ & \\
\hline Rathakrishnan 2008 & 16 & 3 & 5 & 81 & $0.76[0.53,0.92]$ & $0.96[0.90,0.99]$ & $\rightarrow-$ & $\rightarrow$ \\
\hline Tsivgoulis 2007 & 34 & 5 & 9 & 84 & $0.79[0.64,0.90]$ & $0.94[0.87,0.98]$ & $\rightarrow-$ & $\rightarrow$ \\
\hline Tsivgoulis 2008 & 17 & 6 & 81 & 182 & $0.68[0.46,0.85]$ & $0.97[0.93,0.99]$ & 1 & $=$ \\
\hline Wada 2002 & 3 & 1 & 0 & 86 & $1.00(0.29,1.00)$ & $0.99(0.94,1.00)$ & & $\Rightarrow$ \\
\hline
\end{tabular}

Fig. 2 Forest plot. Estimated diagnostic accuracy of transcranial ultrasonography in detecting steno-occlusive lesions in acute stroke population

hyperechoic appearance of MCA with no Doppler signal or waveform suggestive of a large occlusion, similar to hyperdense MCA on CT. Occlusion of one or two terminal branches of the MCA presenting as lacunar stroke has been reported to be "insufficient" to cause changes detectable with TCD [47].

Diagnostic accuracy values for the detection of cerebral steno-occlusive lesions in comparison to angiography as the reference test (CTA [23, 25, 28, 33, 38], CTA/MRA/ DSA [39], DSA [40]) are presented in the forest plot (Fig. 2) and SROC plot (Fig. 3).

In general, there was a good correlation between TCD and reference standard findings in the acute ischaemic stroke population, with the sensitivity ranging from 68 to $100 \%$ and the specificity $78-99 \%$. The area under the SROC curve was 0.91 . However, TCD appeared to be more sensitive in detecting MCA steno-occlusive lesions when compared to assessment of the posterior cerebral circulation but was less specific (Additional file 4) [33, 38],

Transcranial ultrasonography can also provide evidence of intracranial haemorrhage in some cases. Acute haemorrhagic foci are usually seen on ultrasound images as homogenous hyperechogenic structures that are well distinguished from surrounding tissues. Transcranial ultrasound has been shown to detect $52.9 \%$ of haemorrhagic foci in deep cerebral structures, such as basal ganglia, whereas cortico-subcortical lobar lesions were missed in about $67 \%$ of cases, mainly in the temporal and frontal lobes [30, 34, 44], There was a good correlation between the volumes of haemorrhagic foci measured by transcranial ultrasound and CT [31, 42, 44]. It revealed haemorrhagic foci with volumes as small as $0.47 \mathrm{~mm}^{3}$ and as large as $234 \mathrm{~mm}^{3}$ [44].

Although transcranial ultrasonography has been shown to identify haemorrhage in supratentorial and infratentorial locations, and intraventricular haemorrhages [42], echogenicity of haemorrhage appears to be similar to that of the cerebellar tentorium and calcifications within choroid plexuses. Cerebral tumours, vascular malformations and cerebral microangiopathies may also appear as hyperechogenic areas mimicking haemorrhagic lesions $[44,48]$.

Transcranial ultrasound has been demonstrated to be a useful diagnostic tool in detecting midline shift in space-occupying haemorrhagic or ischaemic stroke lesions [42]. There was a good consistency between the studies in the technique used to measure midline shift as a deviation from the presumed midline ipsilaterally and contralaterally to the focus as a distance between the source of the ultrasound beam and the centre of the third ventricle.

There was an excellent correlation between transcranial ultrasonography and CT measurements of the midline shift with a coefficient of about 0.9 [26, 36, 37, 44]. Midline shift has been reported to be the most sensitive indicator of intracranial haemorrhage with a significant correlation between haemorrhagic focus volume and midline shift [37, 44].

\section{Ultrasound window availability}

One of the technical limitations of transcranial ultrasonography is the inability to visualise brain structures of interest in patients who have an inadequate temporal bone acoustic window.

The average incidence of temporal acoustic window failure in the included studies was $19.7 \%$, which led to false negative results in a number of cases [27]. Notably, in the study by Matsumoto et al. [31], the majority of patients were Asian, and there was a higher reported rate of temporal window failure of $56.3 \%$ of cases (27/48).

\section{Risk of bias assessment across studies}

Since only seven papers presented study results reporting quantitative values of the diagnostic accuracy of the test, publication bias assessment illustrating the possibility of selective publication of small studies with positive results was not performed. 


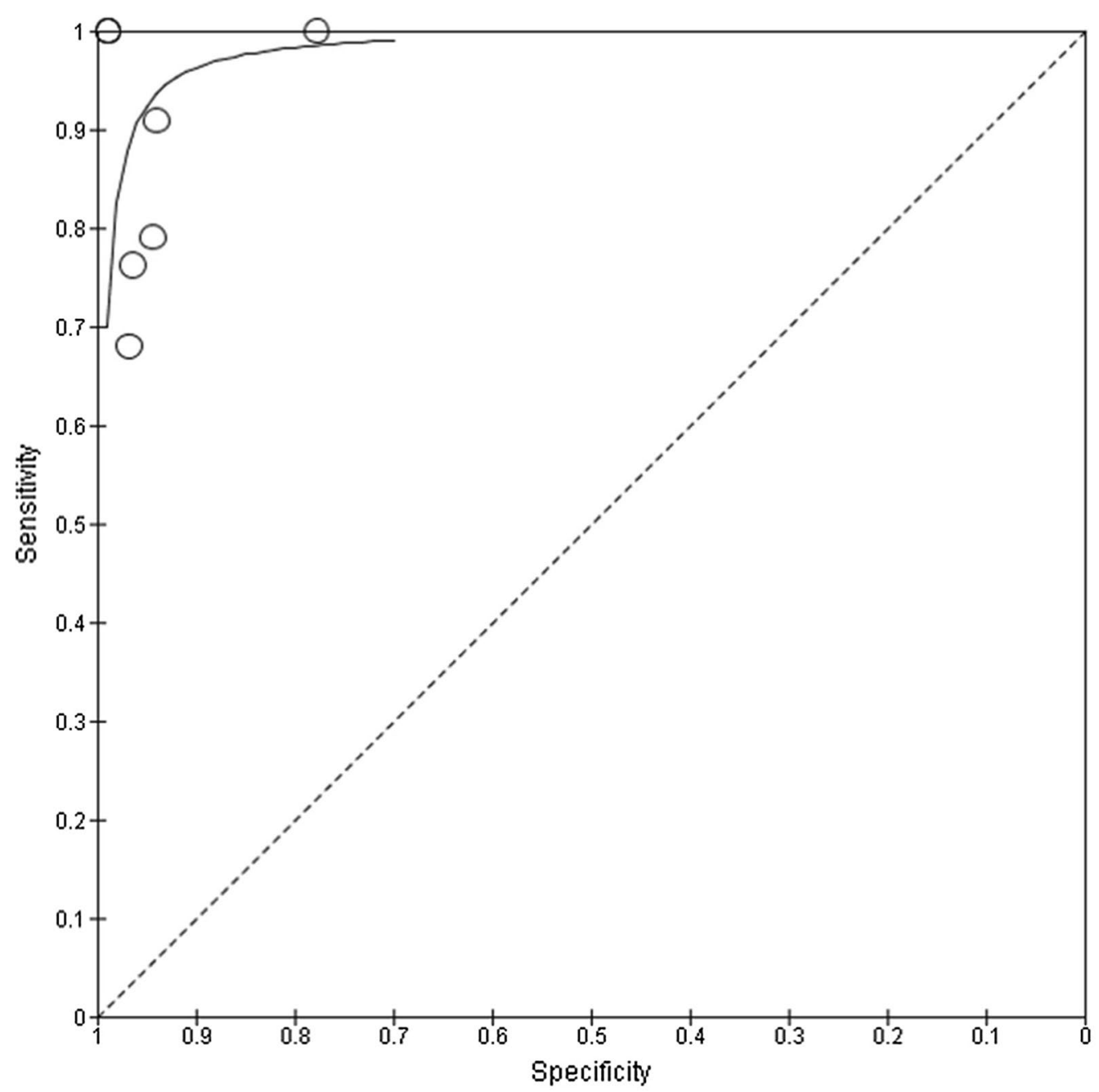

Fig. 3 Summary receiver operating characteristic plot. Each circle represents the sensitivity and specificity estimate from one study

\section{Discussion}

There is a substantial number of published diagnostic accuracy evaluations of transcranial ultrasonography in acute stroke population. 27 English-language studies that compared ultrasonography with other conventional imaging tools within $72 \mathrm{~h}$ of stroke symptom onset and with not more than $24 \mathrm{~h}$ ' time difference between the index and reference tests were identified.

Different types of transcranial ultrasonography were used in the studies selected for the final analysis. TCD appeared to be the most commonly employed techniques, which could be explained by the fact that more than $80 \%$ of the papers aimed to evaluate cerebral blood flow and to detect signs of acute occlusion and/or stenosis.

Seventeen studies included in the current review evaluated diagnostic value of TCD in identifying patients with acute vessel occlusion. Thus, absent or diminished Doppler signal in the MCA mainstem is suggestive of its occlusion [38]. Asymmetry index equal or higher than $21 \%$ is considered indicative of a large branch or multiple branch occlusion. It is less likely to be of diagnostic value in lacunar stroke cases as blood flow abnormalities caused by MCA terminal branch occlusion might not be detected with TCD $[21,29,47]$. A finding of hyperechoic MCA signal is comparable to a hyperdense MCA on CT and suggestive of a large vessel occlusion [43].

The sensitivity and specificity for detecting stenoocclusive lesions in cerebral arteries were $68-100 \%$ and 78-99\%, respectively, with the highest specificity for MCA lesions. These findings could potentially be used for the selection of subjects with LVO for direct transfer to an endovascular centre. Its application for detection of distal MCA stenosis [46] and steno-occlusive lesions in the posterior cerebral circulation is, however, limited. 
This might be explained by a suboptimal angle of insonation which may lead to ambiguous findings [23, 24, 29, 33, 38]. This limitation can be potentially overcome by obtaining angle-corrected flow velocities [24].

There was a significant variation in diagnostic criteria used to detect acute steno-occlusive lesions. The lack of any approved diagnostic protocol for assessing cerebral artery occlusion and stenosis represents a big challenge as the results of studies are not absolutely compatible. There are a few recognised limitations of TCD, such as an inability to visualise anatomical landmarks, and as a consequence, a risk of inaccurate classification of specific blood vessels. Identification of blood vessels usually proceeds on the basis of indirect parameters, in particular: depth of insonation, flow direction and transducer orientation and position.

Differentiation between haemorrhagic and ischemic stroke is an important step in determining patient pathways. Transcranial ultrasonography can be used in some cases to detect intraparenchymal bleeds located in the brain structures that can be easily visualised with ultrasonographic examination, such as the basal ganglia. However, imaging of cortical regions, specifically frontal and parietal lobes, is largely restricted by the low spatial resolution of transcranial ultrasonography in these areas. It has also been reported that trace amounts of blood in the ventricular system may be missed, specifically in the occipital horn of the lateral ventricle [17]. Furthermore, some pathological lesions within the brain structures, such as calcifications, tumours, and vascular malformation can mimic the appearance of intracranial haemorrhage, and therefore may lead to false positive ultrasonographic findings [44, 48]. Midline shift might be used as an indirect parameter suggestive of increased intracranial pressure due to space-occupying stroke lesion $[26,42]$.

Our search also did not identify any eligible papers assessing diagnostic accuracy of transcranial ultrasonography in detecting signs of spontaneous SAH that have been described elsewhere as a hyperechogenic signal in the basal cisterns [49]. Studies evaluating diagnostic accuracy of transcranial ultrasonography for the detection of vasospasm after SAH were excluded from the current review.

Transcranial ultrasound is a simple, non-invasive and affordable diagnostic tool that can be repeated as many times as required at the patient's bedside and in spacerestricted environments, such as ambulances $[31,44,50$, 51]. It takes on average 4.3-13.6 min for the complete examination of cerebral vessels $[27,41]$. It can provide useful information complementary to clinical assessment, serving as a "stethoscope for the brain" [28]. A fasttrack protocol for contrast-enhanced duplex ultrasound assessment in the Emergency Department has been suggested by Connolly et al. [52]. They demonstrated that the mean time to complete the ultrasound examination reduced on average by 1 min $46 \mathrm{~s}$ if performed by more experienced sonographers. As demonstrated previously $[50,51,53]$, transcranial ultrasound images can be transferred for expert interpretation from remotely supported ambulances in remote and rural areas.

There are, however, a few limitations that restrict its use from being more widespread: namely the operatordependent nature of interpretation, and the possibility of patients having inadequate temporal acoustic windows. Temporal bone acoustic window failure has been reported in $8-29 \%$ of the general population, and is more commonly seen in women over the age of 50 years and the Asian population [54, 55]. Contrast-enhanced transcranial ultrasonography may be considered as a possible solution, since it has been shown to provide a better visualisation compared with non-enhanced examination [41]. Further research is required for technique optimization of transcranial ultrasonography with the potential application of lower frequency, which may provide greater penetration through the skull into the brain.

Early definitive imaging in patients suspected of having suffered an acute stroke is desirable. However, rapid performance of a CT or angiography is unrealistic in many remote regions of the world requiring expensive and sometimes hazardous transfers to hospital. The gathered evidence suggests that transcranial ultrasound assessment alone currently does not allow an accurate diagnosis particularly in haemorrhagic stroke cases which is the main requirement for this technique to be used in the reliable pre-hospital triage process. However, its diagnostic accuracy might be improved by adding clinical assessment [5] to justify transfer to hospital associated with high costs and risks.

\section{Limitations and bias}

Given the highly specific nature of the literature search, almost all of the relevant literature available is believed to have been retrieved. However, papers in languages other than English were not included. Some papers reporting negative results in their evaluation of diagnostic accuracy of transcranial ultrasonography in acute stroke were not included, since they did not meet our inclusion criteria $[18,56]$. Thus, Kamal et al. [18] studied cerebral artery stenosis in stroke patients from a South Asian population, and concluded that TCD has poor sensitivity for detection of arterial stenosis when compared to MRA. This study was not included in the qualitative analysis because of the unclear time interval between stroke onset and examinations and between index and reference tests. Suwanwela et al. [56] reported that TCD should not be 
used as a screening tool for identifying stenotic lesions in MCA territory stroke since a normal TCD examination does not exclude stenosis of the distal MCA branches. This paper was excluded from the final analysis as the time interval between TCD and CTA could exceed $24 \mathrm{~h}$. However, none of the above studies reported findings that would contradict the results of the other reviewed papers.

Three studies that employed case-control methodology to compare transcranial ultrasound findings in patients with acute stroke and a healthy age-matched population were identified. Case-control studies have been shown to report two- or threefold higher estimates of diagnostic accuracy when compared to single series studies (spectrum bias) [57].

Most of the studies were relatively small, with the number of recruited patients being less than 100, and all were conducted in in-hospital settings. This might indicate potential selection bias. The present systematic review was not restricted to studies recruiting consecutive participants, however, a protocol which allows researchers to select individuals for inclusion in the study may also have selection bias.

One source of potential biases in the methodology relating to the reference test involved lack of blinding of investigators performing or interpreting results. This might affect the interpretation of the results potentially leading to either over- or underestimated performance of the reference test.

A time interval of not more than $24 \mathrm{~h}$ between the index and reference imaging to ensure minimal discrepancy between studies due to evolution of changes as a result of hypoxia or thrombus propagation, dissolution, or reocclusion $[28,38]$ was one of the inclusion criteria for the present systematic review. Therefore, this source of bias was not identified in any of the papers. However, the timing of the diagnostic tests was often not specifically reported as within $24 \mathrm{~h}$ but, for instance, "on admission".

Some potential biases were identified indicated by inconsistent reporting of important methodological details, such as level of expertise of the person performing the investigation with transcranial ultrasonography and blindness of investigators performing and/or interpreting reference and index tests. In the majority of the studies, ultrasonography investigation was performed by a specialist, reducing the risk of potential diagnostic bias in these cases. It is important to ensure that operators performing or interpreting transcranial ultrasound scans have specialist knowledge and adequate experience in the field if their assessment results are to be used to guide management of acute stroke patients. Inexperienced transcranial ultrasound users might potentially misinterpret findings leading to higher rates of false results and consequent wrong management decisions.

It appears that in most studies the index and reference tests were interpreted independently, without knowledge of the other's findings. About 37\% (10/27) of reviewed studies included fewer than $80 \%$ of the participants in the analysis of outcomes and results which may introduce bias in the studies if there is some systematic difference between the excluded participants and those included in the final analysis.

In some studies, several imaging modalities were used as reference tests $[21,25,27,39]$. The choice of imaging modality in acute stroke is guided by different factors, including suspected type of stroke, the time from symptom onset, contraindications for specific diagnostic tool, etc. However, such variability across the study population might lead to misinterpretation of the diagnostic accuracy of the index test.

Currently, a sector (cardiac) probe is most commonly used for the purposes of transcranial ultrasonography, since it has a small footprint and allows scanning at low frequency (2-4 MHz). However, it does not allow a continuous satisfactory visualisation of brain structures within the acoustic window during the scanning due to its poor conformity with temporal bone anatomy. Future work is needed to develop a probe that would be anatomically suitable specifically for transcranial scanning and allows imaging at angles that would allow better coverage of the brain.

\section{Conclusions}

This systematic review demonstrates that transcranial ultrasonography might potentially be used for the selection of subjects with acute LVO, to help streamline patient care and allow direct transfer to specialised endovascular centres. It can also assist in detecting haemorrhagic lesions in some cases, however, its applicability here is largely restricted. Further work is needed to establish a formal diagnostic protocol and criteria for detecting signs of acute stroke, to ensure consistency in reporting results. Additional research should optimize the scanning technique at lower frequency with potential for construction of a dedicated probe that would be compatible with anatomical shape of the temporal bone windows. Future work is also required to demonstrate whether this diagnostic approach, possibly combined with clinical assessment, could be used at the pre-hospital stage to justify direct transfer to a regional thrombectomy centre in suitable cases. 


\section{Supplementary information}

Supplementary information accompanies this paper at https://doi. org/10.1186/s13089-019-0143-6.

Additional file 1. Search strategy.

Additional file 2. Criteria for the diagnosis of arterial stenosis in individual studies.

Additional file 3. Criteria for the diagnosis of arterial occlusion in individual studies.

Additional file 4. The accuracy parameters of transcranial ultrasonography for detecting cerebral arterial stenosis or occlusion in individual studies.

\section{Abbreviations}

CT: computed tomography; CTA: computed tomography angiography; DSA: digital subtraction angiography; FN: false negative value; FP: false positive value; LVO: large vessel occlusion; MCA: middle cerebral artery; MRA: magnetic resonance angiography; MRI: magnetic resonance imaging; MT: mechanical thrombectomy; SAH: subarachnoid haemorrhage; SROC: summary receiver operating characteristics; TCD: transcranial Doppler sonography; TN: true negative value; TP: true positive value.

\section{Acknowledgements}

This work is part of a Ph.D. project supported by the University of Aberdeen's Elphinstone Scholarship Programme. LE's work was funded by the European Space Agency SatCare grant. The authors would like to thank Dr. Lorenzo Bandieri for the help with assessing full text of articles for eligibility for the current review.

\section{Authors' contributions}

All four authors were involved in the conceptualisation and development of the methodology. DA performed a complete literature search. Assessment of full text of articles for eligibility and data extraction was performed by DA and LE. DA performed a formal analysis of the collected data and writing of the original draft (including all tables and figures). DA finalised all drafts in consultation with LE, AM and PW. LE, AM and PW reviewed the manuscript. All authors read and approved the final manuscript.

\section{Funding}

No specific funding was received for this work.

\section{Availability of data and materials \\ Not applicable.}

Ethics approval and consent to participate

Not applicable.

\section{Consent for publication}

Not applicable.

\section{Competing interests}

The authors declare that they have no competing interests.

\section{Glossary}

Transcranial brightness mode (b-mode) sonography

Transcranial Doppler sonography (TCD)
Application of low-frequency ultrasound wave through a thin skull bone to enable a grey-scale visualisation of cerebral structures [58].

A pulsed Doppler system with low transmitter frequency for recording blood flow velocities from basal cerebral arteries on the basis of indirect parameters: the depth of the sample volume, the position of the transducer, and the flow direction [59].
Transcranial A method of ultrasonography that enables the colour-coded duplex visualisation of the basal cerebral arteries by colour sonography (TCCS) coding of blood flow velocity; a combination of b-mode and Doppler ultrasonography [58].

\section{Author details}

${ }^{1}$ Centre for Rural Health, University of Aberdeen, Old Perth Road, Inverness IV2 $3 \mathrm{JH}$, UK. ${ }^{2}$ Department of Stroke and Rehabilitation Medicine, Raigmore Hospital, NHS Highland, Inverness IV2 3UJ, UK.

Received: 2 May 2019 Accepted: 4 October 2019

Published online: 22 October 2019

\section{References}

1. Adamson J, Beswick A, Ebrahim S (2004) Is stroke the most common cause of disability? J Stroke Cerebrovasc Dis 13:171-177

2. Goyal M, Menon BK, van Zwam WH, Dippel DWJ, Mitchell PJ, Demchuk AM et al (2016) Endovascular thrombectomy after large-vessel ischaemic stroke: a meta-analysis of individual patient data from five randomised trials. Lancet 387:1723-1731

3. Saver JL, Goyal M, van der Lugt A, Menon BK, Majoie CBLM, Dippel DW et al (2016) Time to treatment with endovascular thrombectomy and outcomes from ischemic stroke: a meta-analysis. JAMA 316:1279-1288

4. Saver JL, Fonarow GC, Smith EE, Reeves MJ, Grau-Sepulveda MV, Pan W et al (2013) Time to treatment with intravenous tissue plasminogen activator and outcome from acute ischemic stroke. JAMA 309:2480-2488

5. Turc G, Maier B, Naggara O, Seners P, Isabel C, Tisserand M et al (2016) Clinical scales do not reliably identify acute ischemic stroke patients with large-artery occlusion. Stroke 47:1466-1472

6. Martinez-Sanchez P, Serena J, Alexandrov AV, Fuentes B, FernandezDominguez J, Diez-Tejedor E (2009) Update on ultrasound techniques for the diagnosis of cerebral ischemia. Cerebrovasc Dis 27(Suppl 1):9-18

7. Whiting PF, Rutjes AWS, Westwood ME, Mallett S, Deeks JJ, Reitsma JB et al (2011) QUADAS-2: a revised tool for the quality assessment of diagnostic accuracy studies. Ann Intern Med 155:529-536

8. Macaskill P, Gatsonis C, Deeks JJ, Harbord RM, Takwoingi Y (2013) Chapter 10: Analysing and presenting results. In: Deeks JJ, Bossuyt PM, Gatsonis C (eds) Cochrane handbook for systematic reviews of diagnostic test accuracy Version 100. The Cochrane Collaboration. https:// srdta.cochrane.org

9. Schlachetzki F, Herzberg M, Holscher T, Ertl M, Zimmermann M, Ittner KP et al (2012) Transcranial ultrasound from diagnosis to early stroke treatment: part 2: prehospital neurosonography in patients with acute stroke: the Regensburg stroke mobile project. Cerebrovasc Dis 33:262-271

10. Saqqur M, Hill MD, Alexandrov AV, Roy J, Schebel M, Krol A et al (2006) Derivation of power M-mode transcranial Doppler criteria for angiographic proven MCA occlusion. J Neuroimaging 16:323-328

11. Ogata T, Kimura K, Nakajima M, Naritomi H, Minematsu K (2005) Diagnosis of middle cerebral artery occlusive lesions with contrastenhanced transcranial color-coded real-time sonography in acute stroke. Neuroradiology 47:256-262

12. Motuel J, Biette I, Srairi M, Mrozek S, Kurrek MM, Chaynes P et al (2014) Assessment of brain midline shift using sonography in neurosurgical ICU patients. Crit Care 18:676

13. Herzberg M, Boy S, Holscher T, Ertl M, Zimmermann M, Ittner K-P et al (2014) Prehospital stroke diagnostics based on neurological examination and transcranial ultrasound. Crit Ultrasound J 6:3

14. de Bray JM, Missoum A, Dubas F, Emile J, Lhoste P (1997) Detection of vertebrobasilar intracranial stenoses: transcranial Doppler sonography versus angiography. J Ultrasound Med 16:213-218

15. Bertram M, Khoja W, Ringleb P, Schwab S (2000) Transcranial colourcoded sonography for the bedside evaluation of mass effect after stroke. Eur J Neurol 7:639-646 
16. Alexandrov AV, Bladin CF, Norris JW (1994) Intracranial blood flow velocities in acute ischemic stroke. Stroke 25:1378-1383

17. Mäurer M, Shambal S, Berg D, Woydt M, Hofmann E, Georgiadis D et al (1998) Differentiation between intracerebral hemorrhage and ischemic stroke by transcranial color-coded duplex-sonography. Stroke 29:2563-2567

18. Kamal AK, Rehman H, Mustafa N, Ahmed B, Jan M, Wadivalla F et al (2015) Diagnostic TCD for intracranial stenosis in acute stroke patients: experience from a tertiary care stroke center in Karachi, Pakistan. BMC Res Notes 8:341

19. Akopov S, Whitman GT (2002) Hemodynamic studies in early ischemic stroke: serial transcranial Doppler and magnetic resonance angiography evaluation. Stroke 33:1274-1279

20. Panerai RB, Jara JL, Saeed NP, Horsfield MA, Robinson TG (2016) Dynamic cerebral autoregulation following acute ischaemic stroke: comparison of transcranial Doppler and magnetic resonance imaging techniques. J Cereb Blood Flow Metab 36:2194-2202

21. Viola S, Tenaglia MG, De Leonardis E, Aquilone L, Gambi D (1993) Acute hemispheric stroke: correlation between three-dimensional transcranial Doppler, MR-angiography, CT and clinical findings. Ital J Neurol Sci 14:225-232

22. Meader N, King K, Llewellyn A, Norman G, Brown J, Rodgers M et al (2014) A checklist designed to aid consistency and reproducibility of GRADE assessments: development and pilot validation. Syst Rev 3:82

23. Bar M, Skoloudik D, Roubec M, Hradilek P, Chmelova J, Czerny D et al (2010) Transcranial duplex sonography and CT angiography in acute stroke patients. J Neuroimaging 20:240-245

24. Boddu DB, Sharma VK, Bandaru VCSS, Jyotsna Y, Padmaja D, Suvarna A et al (2011) Validation of transcranial Doppler with magnetic resonance angiography in acute cerebral ischemia. J Neuroimaging 21:e34-e40

25. Brunser AM, Lavados PM, Hoppe A, Lopez J, Valenzuela M, Rivas R (2009) Accuracy of transcranial Doppler compared with CT angiography in diagnosing arterial obstructions in acute ischemic strokes. Stroke 40:2037-2041

26. Gerriets T, Stolz E, Konig S, Babacan S, Fiss I, Jauss M et al (2001) Sonographic monitoring of midline shift in space-occupying stroke: an early outcome predictor. Stroke 32:442-447

27. Gerriets T, Goertler M, Stolz E, Postert T, Sliwka U, Schlachetzki F et al (2002) Feasibility and validity of transcranial duplex sonography in patients with acute stroke. J Neurol Neurosurg Psychiatry 73:17-20

28. Guan J, Zhou Q, Ouyang H, Zhang S, Lu Z (2013) The diagnostic accuracy of TCD for intracranial arterial stenosis/occlusion in patients with acute ischemic stroke: the importance of time interval between detection of TCD and CTA. Neurol Res 35:930-936

29. Kenton AR, Martin PJ, Abbott RJ, Moody AR (1997) Comparison of transcranial color-coded sonography and magnetic resonance angiography in acute stroke. Stroke 28:1601-1606

30. Kern R, Kablau M, Sallustio F, Fatar M, Stroick M, Hennerici MG et al (2008) Improved detection of intracerebral hemorrhage with transcranial ultrasound perfusion imaging. Cerebrovasc Dis 26:277-283

31. Matsumoto N, Kimura K, Iguchi Y, Aoki J (2011) Evaluation of cerebral hemorrhage volume using transcranial color-coded duplex sonography. J Neuroimaging 21:355-358

32. Nasr N, Ssi-Yan-Kai G, Guidolin B, Bonneville F, Larrue V (2013) Transcranial color-coded sonography to predict recurrent transient ischaemic attack/ stroke. Eur J Neurol 20:1212-1217

33. Rathakrishnan R, Berne YI, Quek KK, Hong CS, Ong BK, Chan BP et al (2008) Validation of transcranial Doppler with CT angiography in cerebral ischaemia: a preliminary pilot study in Singapore. Ann Acad Med Singapore 37:402-405

34. Seidel G, Cangur H, Albers T, Meyer-Wiethe K (2005) Transcranial sonographic monitoring of hemorrhagic transformation in patients with acute middle cerebral artery infarction. J Neuroimaging 15:326-330

35. Seidel G, Cangur H, Albers T, Burgemeister A, Meyer-Wiethe K (2009) Sonographic evaluation of hemorrhagic transformation and arterial recanalization in acute hemispheric ischemic stroke. Stroke 40:119-123

36. Stolz E, Gerriets T, Fiss I, Babacan SS, Seidel G, Kaps M (1999) Comparison of transcranial color-coded duplex sonography and cranial CT measurements for determining third ventricle midline shift in space-occupying stroke. Am J Neuroradiol 20:1567-1571
37. Tang SC, Huang SJ, Jeng JS, Yip PK (2006) Third ventricle midline shift due to spontaneous supratentorial intracerebral hemorrhage evaluated by transcranial color-coded sonography. J Ultrasound Med 25:203-209

38. Tsivgoulis G, Sharma VK, Lao AY, Malkoff MD, Alexandrov AV (2007) Validation of transcranial Doppler with computed tomography angiography in acute cerebral ischemia. Stroke 38:1245-1249

39. Tsivgoulis G, Sharma VK, Hoover SL, Lao AY, Ardelt AA, Malkoff MD et al (2008) Applications and advantages of power motion-mode Doppler in acute posterior circulation cerebral ischemia. Stroke 39:1197-1204

40. Wada K, Kimura K, Minematsu K, Yasaka M, Uchino M, Yamaguchi T (2002) Combined carotid and transcranial color-coded sonography in acute ischemic stroke. Eur J Ultrasound 15:101-108

41. Goertler M, Kross R, Baeumer M, Jost S, Grote R, Weber S et al (1998) Diagnostic impact and prognostic relevance of early contrast-enhanced transcranial color-coded duplex sonography in acute stroke. Stroke 29:955-962

42. Ovesen C, Christensen AF, Krieger DW, Rosenbaum S, Havsteen I, Christensen H (2014) Time course of early postadmission hematoma expansion in spontaneous intracerebral hemorrhage. Stroke 45:994-999

43. Kadimi S, Terry JB, Gomez CR (2000) Hyperechoic middle cerebral artery: acute occlusion detected by transcranial duplex ultrasonography. J Neuroimaging 10:228-230

44. Kukulska-Pawluczuk B, Ksiazkiewicz B, Nowaczewska M (2012) Imaging of spontaneous intracerebral hemorrhages by means of transcranial colorcoded sonography. Eur J Radiol 81:1253-1258

45. Leanyvari Z, Vastagh I, Fulesdi B, Szirmai I, Lengyel A, Csiba L et al (2002) Computed tomographic and transcranial Doppler sonographic findings in acute and subacute phases of middle cerebral artery strokes. J Clin Ultrasound 30:33-37

46. Zubkov AY, Uschmann H, Rabinstein AA (2008) Rate of arterial occlusion in patients with acute ischemic stroke. Neurol Res 30:835-838

47. Zanette EM, Fieschi C, Bozzao L, Roberti C, Toni D, Argentino C et al (1989) Comparison of cerebral angiography and transcranial Doppler sonography in acute stroke. Stroke 20:899-903

48. Seidel G, Gerriets T, Kaps M, Missler U (1996) Dislocation of the third ventricle due to space-occupying stroke evaluated by transcranial duplex sonography. J Neuroimaging 6:227-230

49. Olatunji RB, Ogbole Gl, Atalabi OM, Adeyinka AO, Lagunju I, Oyinlade A et al (2015) Role of transcranial colour-coded duplex sonography in stroke management—review article. West African J Ultrasound 16:33-42

50. Eadie L, Mulhern J, Regan L, Mort A, Shannon H, Macaden A et al (2018) Remotely supported prehospital ultrasound: a feasibility study of realtime image transmission and expert guidance to aid diagnosis in remote and rural communities. J Telemed Telecare 24:616-622

51. Eadie L, Regan L, Mort A, Shannon H, Walker J, MacAden A et al (2015) Telestroke assessment on the move: prehospital streamlining of patient pathways. Stroke 46:e38-e40

52. Connolly F, Rohl J-E, Guthke C, Wengert O, Valdueza JM, Schreiber SJ (2019) Emergency room use of "fast-track" ultrasound in acute stroke: an observational study. Ultrasound Med Biol 45:1103-1111

53. Mort A, Eadie L, Regan L, Macaden A, Heaney D, Bouamrane MM et al (2016) Combining transcranial ultrasound with intelligent communication methods to enhance the remote assessment and management of stroke patients: framework for a technology demonstrator. Health Inform J 22:691-701

54. Maeda H, Matsumoto M, Handa N, Hougaku H, Ogawa S, Itoh T et al (1993) Reactivity of cerebral blood flow to carbon dioxide in various types of ischemic cerebrovascular disease: evaluation by the transcranial Doppler method. Stroke 24:670-675

55. Marinoni M, Ginanneschi A, Forleo P, Amaducci L (1997) Technical limits in transcranial Doppler recording: inadequate acoustic windows. Ultrasound Med Biol 23:1275-1277

56. Suwanwela NC, Suwanwela N, Phanthumchinda K (2000) Comparison of transcranial Doppler ultrasound and computed tomography angiography in symptomatic middle cerebral artery stenosis. Australas Radiol 44:174-177

57. Whiting P, Rutjes AWS, Reitsma JB, Glas AS, Bossuyt PMM, Kleijnen J (2004) Sources of variation and bias in studies of diagnostic accuracy: a systematic review. Ann Intern Med 140:189-202 
58. Bartels E (2012) Transcranial color-coded duplex ultrasonography in routine cerebrovascular diagnostics. Perspect Med 1-12:325-330

59. Arnolds BJ, von Reutern G-M (1986) Transcranial Dopplersonography. Examination technique and normal reference values. Ultrasound Med Biol 12:115-123

\section{Publisher's Note}

Springer Nature remains neutral with regard to jurisdictional claims in published maps and institutional affiliations.
Submit your manuscript to a SpringerOpen ${ }^{\odot}$ journal and benefit from:

- Convenient online submission

- Rigorous peer review

- Open access: articles freely available online

- High visibility within the field

- Retaining the copyright to your article

Submit your next manuscript at $\boldsymbol{\nabla}$ springeropen.com 